\title{
Questioning Searle's Explanation of Unconscious Mental State
}

\author{
Yuhua Zhou', Pengzhi Hu² \\ ${ }^{1}$ Zhejiang Yuexiu University, Shaoxing, China \\ ${ }^{2}$ Shaoxing University, Shaoxing, China \\ Email: 20131050@zyufl.edu.cn
}

How to cite this paper: Zhou, Y.H. and Hu, P.Z. (2021) Questioning Searle's Explanation of Unconscious Mental State. Open Access Library Journal, 8: e7581.

https://doi.org/10.4236/oalib.1107581

Received: May 25, 2021

Accepted: July 4, 2021

Published: July 7, 2021

Copyright () 2021 by author(s) and Open Access Library Inc.

This work is licensed under the Creative Commons Attribution International License (CC BY 4.0).

http://creativecommons.org/licenses/by/4.0/

\begin{abstract}
Upholding the principle of Biological Naturalism, John Searle requires that explanation of mental phenomenon must be coherent with findings in scientific studies. To meet the requirement of coherence, Searle puts forward his "connection principle". This paper contends that his requirement is not well met in his own study of unconscious mental states. Firstly, his classification of unconscious mental states is not reasonable. For example, he has classified "knowledge of language" into deep unconscious mental state, which in nature belongs to nonconscious mental state. The classification is not in accord with findings in linguistic studies, nor with linguistic practice. Secondly, his explanation for the unconscious mental process is doubtful. Based on his "connection principle", unconscious mental state is nothing but potential conscious mental state and therefore the processing of unconscious mental activity should resemble that of conscious mental activity. But this explanation is not coherent with findings in cognitive science. The paper concludes that Searle fails in his requirement of coherence.
\end{abstract}

\section{Subject Areas}

Linguistics, Psychology

\section{Keywords}

Unconscious Mental State, Connection Principle, Biological Naturalism, Coherence

\section{1. 引言}

对无意识现象的认识可追溯至柏拉图, 其后笛卡尔、莱布尼兹、康德等 理性主义者在讨论心身关系时都有所涉及。真正将无意识作为研究对象提出 
的是弗洛伊德。弗洛伊德对于无意识的论述主要基于心理学视角, 其无意识 理论可总结为以下三个要点: 第一, 所有意识状态就其本身而言都是无意识 的, 意识心智状态和无意识心智状态之间的关系可最终还原为能量之间的变 换关系 [1], 即无意识心智状态获得足够能量, 即可被 “感知” , 渗透到意识 中来; 第二, 无意识心智状态在本体论上是主观的, 虽然无意识介于心理和 生理之间, 但其核心是本能表征一一观念 [1]; 第三, 无意识心智状态与主体 的行为之间存在直接的因果关系, 弗洛伊德认为无论是本能还是欲望, 它们 均以无意识心智状态存在, 它们才是支配生命活动的最基本法则和最基本动 力。

弗洛伊德的无意识理论一直备受争议, 批评者甚至指出其学说既不科学 也不具有治疗作用, 有欺世盗名之嫌。现有批评多从心理学入手, 因此塞尔 从心智哲学角度的批评可谓独树一帜。

塞尔将自己的立场概括为 “生物学自然主义” (Biological Naturalism), 其 核心内容为 “联结原则” (Connection Principle), 表示 “无意识心智状态蕴涵 了意识的可进入性。我们没有无意识状态, 除非它是潜在地有意识的” [2]。 联结原则之下的无意识心智状态具有以下三个特征, 它们与弗洛伊德无意识 理论中的三个要点针锋相对。第一, “我们把无意识心智状态的概念理解为 只不过是意识的可能内容。” [2]塞尔将意识状态视为心智状态的主导因素, 无意识心智状态只不过是潜在的意识状态。第二, 就本体论而言, “在状态 是完全无意识的时候, 除了神经生理状态与过程之外, 别无他物。” [2]因此, 无意识心智状态在本体论上是客观的。第三, 无意识心智状态与主体行为之 间因果关系的实现依赖于能够引起意识状态的神经生物学结构。塞尔认为, 我们将无意识的心智状态指派给一个行为能动者的时候, 我们其实是将一个 能够引起意识的神经生物学结构指派给了它, 这一生理结构在该时刻没有引 起意识状态, 是因为它受到压抑或者遇到了障碍[3]。

本文拟从无意识心智状态的分类和加工方式两个方面入手, 并以具体语 言知识为例, 对塞尔的无意识心智状态论点进行质疑。

\section{2. 塞尔对无意识心智状态的分类及其融贯性问题}

塞尔将弗洛伊德无意识理论中存在的问题总结为以下两个方面: “首先, 我不能使他的无意识本体论观点与我们所知道的大脑知识相一致; 其次, 我 不能在感知与意识之间构建出一个融贯版本的类比。” [2]鉴于这两个方面的 问题, 塞尔认为弗洛伊德的无意识理论既无法融于神经生物学解释, 也无法 在解释心智状态时达到内部(意识状态和无意识状态之间)的协调一致。为了 避免弗洛伊德理论中的问题, 实现对无意识心智状态解释上的融贯性, 塞尔 将各种无意识现象分为四种类型[3]:

\section{第一种: 前意识(preconscious mental states)}

解释: 行为主体的脑中存在着一种结构, 使其能够具有以一种有意识的 形式来产生相关状态的能力。

举例：1）在塞尔熟睡时他仍具有 “乔治 - 华盛顿是美国首位总统” 的信 念。 
2) 在塞尔沉浸于心智哲学思考时他仍具有 “乔治 - 华盛顿是美国首位总 统” 的信念。

\section{第二种: 被压抑的无意识(repressed unconscious mental states)}

解释: 行为主体具有在其行为中发挥因果功能的心智状态, 但是其本人 却对该心智状态的运作毫无意识, 甚至会真诚地否认其存在。

举例: 3) 睡眠中无意识的疼痛会使主体移动身体以保护受伤之处。

4) 在被催眠时, 行为主体会去执行相关指令。

\section{第三种: 深层无意识(deep unconscious mental states)}

解释: 行为主体进行操作时所根据的规则, 不仅仅在事实上是无意识的, 而且这些规则甚至不是那类可能成为有意识的东西。

举例: 5) 孩子通过 “无意识地” 运用那些普遍语言所具有的运算规则来 学会一门语言。

6) 孩子通过对涌现到其视网膜上的输入信息做出 “无意识的” 运算操作 而得到视觉感知。

\section{第四种: 非意识(nonconscious mental states)}

解释: 发生在大脑中, 既对于控制我们的心灵生活起到了关键性的作用, 但却又根本不是心智现象的状态

举例: 7) 对于心智现象很重要的位于突触裂缝处的血清素分泌。

8) 脊髓神经系统对于呼吸活动的控制。

相信绝大多数人对于第一种无意识心智状态的存在不会有异议。我们实 际知道的内容要远多于我们即时意识中的内容, 而某些内容我们之所以相信 自己 “知道” 正是因为它们是潜在的意识内容, 可以在需要时被提取并进入 意识状态。对于第四种无意识心智状态, 塞尔的立场是将其视为 “非意识心 智状态” ——不是心智现象而仅是神经生物学现象, 那么解释它就成了神经 科学和生物学的问题, 可以暂时被排除在心智哲学讨论之外。如此在塞尔看 来, 第一类无意识心智状态可归属于(潜在)意识现象加以解释, 第四类无意 识心智状态可归属于神经生物学现象加以解释, 两者均可以实现解释上的融 贯性。

我们再来看第二种无意识心智状态。依据塞尔的分析, 例 3 和例 4 中均 存在着心智状态与主体行为之间的因果关系: 例 3 中疼痛的心智状态是主体 移动躯体的原因, 例 4 更为明显, 主体接收到指令后, 其包含执行指令动机 的心智状态是执行指令行为发生的原因。仔细审视这些因果关系的形成过程, 我们发现即便主体对于自己的心智状态无意识甚至 “真诚地否认” ，但能够 引起外在行为的一定是主体内在的神经生物学结构。就例 3 而言, “即使在 主体并没有感觉到疼痛时, 那种能够产生有意识的疼痛的神经生物学结构同 样也能够引起那旨在避免疼痛的相应行为。” 对此类无意识现象而言, “在 无意识的情况下, 无意识动机的实存模式(即本体论基础)就是那能够引起具 有意识形式的动机的发生、并能够引起对应于该动机的恰当行为发生的神经 生物学结构所具有的实存模式。” [3]简言之, 此类无意识心智状态与具有相 同行为因果功能的意识心智状态同构, 那么很显然, 此类无意识心智状态也 就是潜在的意识心智状态。塞尔由此在解释上合并了第一种无意识心智状态 
和第二种无意识心智状态, 将它们均定性为潜在的意识心智状态, 从而实现 解释上的融贯性。

最后来分析第三种无意识心智状态。对于此类无意识心智状态, 塞尔的 解释简洁且坚决: “根本就没有此类事例, 根本就没有像 “一个深层无意识 的心智状态, 的东西。” [3] 塞尔对这一解释给出的理由是, 这种无意识心智 状态不具有 “意向状态” , 也不可能有 “面相形式” , 那么它们怎么可能会 指向外在事物? 怎么可能会生成行为动机? 又怎么可能会引起主体的具体行 为? 举例来说, 无论是语言运算规则还是视神经系统中的运算操作, 孩子对 它们的习得过程也就是孩子神经系统和生理系统的成长过程, 在此过程中, 看似内在的神经生理过程促成了外在的语言和视觉行为, 但实际上, 这些内 在过程根本不可能引起有意识的意向活动, 也就不可能具有心理学实在性。

因此, 此种无意识心智状态本质上等同于第四种无意识心智状态, 都是 “非 意识”。

塞尔的论点可以小结如下: 人类的神经生物学进程分为两类, 即能够产 生具有意识形式的神经生物学进程, 以及能将其描述为似乎具有意向性的非 意识的神经生物学进程, 上述分类中前意识和被压抑的无意识属于前一类, 深层无意识和非意识则应归属于后一类。那么, 前两者作为潜在意识心智状 态可以通过意识现象得到解释, 后两者作为神经生物状态可以通过相应学科 研究发现得到解释。塞尔至此实现了对无意识心智状态解释的融贯性。

\section{3. 作为无意识心智状态的语言知识}

塞尔将 “语言知识” 视为深层无意识, 并最终归属 “非意识” 。基于塞 尔的解释, “语言知识” 仅指语言理解和生成过程中纯粹的 “内在运算机制”, 它既不包含语言的物理部分——语音和词形, 也不包含语言相关的概念内容 (语义)和实践运用(语用)。很明显, 塞尔对 “语言知识” 的解释和乔姆斯基生 成语言学研究中的 “I-语言” 概念高度重合 [4]。我们将可以想到的有关 “语 言知识” 的特征一一列举并解释如下:

第一, 语言知识是默示的(tacit) [5]。“默示” 指 “意识不可及”。之所 以不使用 “无意识” 一词, 是因为上节刚刚讨论了塞尔对无意识心智状态的 分类, 为了避免有人误将语言知识与上述某种无意识心智状态对号入座, 特 采用生成语言学研究中常用的 “默示”一词。

第二, 语言知识在脑中表征(represented in the brain)。对于知识在脑中的 具体存储形态、加工方式, 现有科学研究尚无法解答, 但无论如何, 这些 “知 识” 必定存在于大脑之中, 必定以某种形态被 “表征” 。塞尔将孩子习得的 母语知识表述为 “运算规则” , 乔姆斯基还进一步认为人类语言官能的核心 正是 “递归运算机制(recursive mechanism)”, 这些均是对语言知识在脑中表 征方式的设想。

第三, 语言知识的加工过程依据规则。此处的 “规则” 不可被狭义地理 解为社会规则或者运算规则, 而应当被宽泛地理解为事物存在与运行的客观 规律。 
第四, 语言知识对言语表达具有因果作用(causally efficacious) [6]。于母 语而言, 处于语言习得不同阶段的儿童语言表达不同; 于外语而言, 处于不 同学习阶段的成人学习者的语言表达也会不同。造成这些 “不同” 的主要原 因正是儿童或者成人对于所学语言知识的掌握程度存在差异。因此, 语言知 识掌握程度与言语表达之间存在因果关系。

第五, 语言知识进入意识状态是可能的但却并不必要。一方面, 经过语 言研究者的共同努力, 我们认识并描述(部分)语言知识是可能的; 另一方面, 语言知识是否得到认识与描述并不会影响语言表达行为。一个合格的语言使 用者完全可能熟练使用语言但却说不出任何语言规则。

\section{4. 对塞尔无意识心智状态解释的质疑}

\section{1. 语言知识可以进入意识吗?}

在塞尔的分类中, 语言知识归属 “似乎具有意向性的非意识的神经生物 学进程” 。塞尔认定语言知识不可能具有心理实在性的主要理由是此种无意 识现象不具有意向状态和面相形式, 所以不可能是潜在的意识状态, 而能否 成为潜在的意识状态构成了能否成为无意识状态的依据。因此, 沿着这一思 路, 如果我们可以证明语言知识具有意向状态和面相形式, 或者其中的任何 一项, 那么塞尔对无意识心智状态的解释无疑就会受到严峻的挑战。

请判断下面这几句话是否合语法:

例 9: 我说英语很流利。

例 10: 很流利, 我说英语。

例 11: 我很流利说英语。

我们的判断是, 例 9、10 合语法, 例 11 不合。进一步询问周边的数十个 人, 包括父母、邻居、学生、同事, 得到的回答与我们的判断基本一致。当 我们进一步询问为什么例 11 不对时, 父母和邻居的回答差不多: “不清楚, 但我们一般不这样说。” 被问到的学生以为老师在考他们, 仔细分辨之后小 心翼翼地解释道: 应该是状语 “很流利” 的位置不对, 汉语中它们一般放在 句首或者句末, 而不是放在句子中间。于是我又追问, 那为什么 “我很卖力 干活” 好像合语法呢? 学生便开始茫然。最有趣的是一位研究句法的同事, 他在给出判断的同时随手抓过纸笔就开始给我演算句法结构。

抛开解释与追问, 仅就判断这一行为而言, 从父母到同事的回答是一致 的。但即便如此, 我们仍认为这些行为本质上不同, 因为回答者在做出回答 行为时所依据的内在心智状态并不相同: 父母和邻居依据的是语言直觉, 学 生依据的是直觉加上有限的语法知识, 而那个同事很明显依据的是专业的语 言知识。这些心智状态无疑源自于回答者脑中的神经生物学结构。这即表明, 虽然外在的回答行为一致, 但内在的神经生物学结构却并不同。塞尔已经将 直觉类语言行为的内在心智状态划归为 “非意识”, 而同时我们可以肯定我 的同事依据专业知识做出的分析与判断过程一定基于意识心智状态, 那么这 两种完全不同的心智状态是如何与相同的外在行为构成因果关系的呢?

在塞尔的解释中, 心智状态引起外在行为是因为它至少具有意向状态和 面相形式。我同事在做出判断时, 他的心智状态明显指向待判断的例句, 意 
向性条件符合; 同时他的意识中一定包含着判断例句时所需的相关语法知识, 面相形式条件也符合。我父母和邻居在做出判断时他们的直觉同样也指向待 判断的例句, 难道这不也应该被认为是意向性的表现吗? 虽然直觉本身不可 名状, 但在判断过程中, 直觉到例句的指向性非常明显, 直觉理应被认为具 有意向性。塞尔对语言知识的解释受到了质疑!

进一步分析, 直觉具有面相形式吗? 回答应该是否定的。我们很难想象 包含明确内容的直觉, 因为一旦直觉的内容被描述, 那么它也就成了具体知 识, 而不再是直觉了。但在塞尔将语言知识定性为 “非意识” 时, 他的论述 中还包括另一个条件: “不仅仅在事实上是无意识的, 而且这些规则甚至不 是那类可能成为有意识的东西。” 不可否认, 对于从不学习语法知识的人来 说, 他们使用了一辈子语言也 “不知道” 自己语言的语法规则是什么, 甚至 他们都不会去想什么是 “语言规则”。然而, 这些人 “不知道” 、“不去想” 并不代表这些语法规则 “不可知” , 我同事的专业知识就是对语法规则的较 好描述。或许还会有人要问: 我们怎么知道这些语法知识就是对脑中实存的 心智状态的准确表述呢? 这个问题可能谁也无法回答。但这个问题同样适用 于所有人类知识, 恰如我们也会问人类已知的自然规则是否如实描述了自然 界一样。人类的知识可能永远只是在其所处阶段所能做出的 “最佳假设” , 语言知识也不例外。那么, 如果我们认定其它领域的认识是知识, 可以进入 主体意识, 那么对语言的认识当然也是知识, 也可以进入主体意识。塞尔的 解释在此应该受到质疑。

\section{2. 语言知识能引起规则遵守行为吗?}

塞尔将规则遵守行为分为两类: 被规则控制的行为(rule-governed behaviour)和被规则描述的行为(rule-described behaviour)。前者如 “靠右行车” , 后者如 “物体下落” 。意识心智状态的规则遵守行为明显是前者。无意识(注 意: 不是非意识)心智状态作为潜在的意识状态, 其规则遵守行为也是前者, 且必须具有如下这些特征：（1）规则的因果功能具有从世界指向规则的符合 方向以及从规则指向世界的因果关系方向; (2) 这些规则必须具有一个面相 形式; (3) 它们必须在意愿中被加以遵守; (4) 它们被遵守的方式是可以接受 不同的解释的; (5) 它们必定在实在时间中被遵守 [3]。

语言知识引起的行为是否具有这 5 个特征呢?

语言知识虽然在脑中以直觉状态存在, 但其内含对语言表达的规则性要 求, 并且这些要求构成了语言表达在语法上恰当与否的直接原因, 因此特征 (1)被确认。基于直觉的语言知识虽有进入意识的可能, 但处于直觉状态时它 并不包含具体内容, 所以不具有面相形式, 特征(2)被否定。在使用自己的母 语时, 我有时会思考该怎么说更好, 但思考一般只涉及语义表达, 很少会涉 及语法结构组织, 母语表达的规则遵守过程自动完成, 特征(3)被否定。语言 使用实践中，母语表达的灵活性随处可见，如 “我请你喝酒”，“你喝酒， 我请”, “喝酒, 我请你”。在不同的语境下这些表达方式均可以得到恰当 解释, 特征(4)被确认。作为实践行为, 语言表达必定发生在具体时空中。特 征(5)被确认。

特征(2)和特征(3)被否定, 基于语言知识的表达行为就必然只能是 “被规 
则描述的行为” 吗? 被规则描述的行为指的是受制于客观规律的行为, 像前 例中的物体下落行为。此类规则遵守行为最大的特点就是客观性: 不会变化、 不会出错。语言规则遵守行为会变化吗? 当然会, 语言使用行为是不断发展 变化的, 以汉语为例, 且不说现代汉语与古代汉语区别明显, 恐怕现在每年 也都会出现一些新表达、新说法。语言规则遵守行为会出错吗? 当然会, 无 论是口语还是书写语言, 语法上的错误并不少见。由此便可以否定语言规则 遵守行为的客观性了吗? 不急, 还有一点需要澄清: 语言规则遵守行为中的 变化和错误到底是如何形成的?

先以二语学习者为例来做一个类比。二语学习者在进行二语学习时所接 触到的语言知识、语法规则无疑都是正确的, 但他们实际所说出的二语表达 和写出的二语语句却存在着不少错误。一般情况下, 语言学研究者将这些错 误归处于一语干扰、二语语法不够熟练以及语境因素影响等。这也就意味着 二语知识的正确性并不能保证二语规则遵守行为的正确性。二语如此, 一语 也是如此, 只不过一语受到的干扰要明显少于二语。举例来说, 一个人高度 紧张或者非常激动时可能会 “语无伦次”, 出现表达错误。但这些表达错误 并不能否定一语者内在语言知识本身是正确的, 否则语言使用实践中就不会 存在众多的话语自我修补现象。

基于语言知识的规则遵守行为会受到干扰而发生变化或者错误, 这显然 不是 “被描述的行为” 所可能出现的情况, 后者也会受到干扰, 但受到干扰 之后通常情况下相应行为将无法发生。所以, 基于语言知识的规则遵守行为 既不属于塞尔的 “被规则控制的行为”, 也不属于其的 “被规则描述的行为”。 塞尔的解释难适用于语言知识。

\section{5. 无意识认知加工: 对塞尔解释的进一步质疑}

讨论无意识心智状态, 自然不能忽视认知科学的研究成果。基于塞尔的 联结原则, 无意识心智状态就是潜在的意识心智状态, 无意识心智状态与主 体行为之间因果关系的实现依赖于能够引起意识状态的神经生物学结构。依 据这一解释, 无意识心智状态的认知加工方式应该与意识心智状态一致。但 这一认识与现有认知科学研究成果相违背。

首先, 无意识认知加工现象的独立性已经获得心理学实验研究的证实。

20 世纪中叶开始对无意识现象的研究已经由理论假设转入了实验验证, 内隐记忆 (implicit memory)和内隐学习(implicit learning) 是最常用的实验路 径。内隐记忆最早施用于对遗忘症患者的研究, 研究发现这些患者对于某些 信息的记忆超出了研究者的预计, 而这些信息并不在有意识记忆要求的范围 之内。内隐学习指 “在一些明明应该无法发生任何学习行为的情况下, 确定 有某种形式的学习自动地发生了” [7], 并且此类学习过程学生一无所知, 学 习的效果也无法通过有意识的测试加以检验 [8]。内隐记忆、内隐学习等领域 的实证研究发现确认了人类无意识认知加工活动的存在, 不仅如此, 它们还 同时证实无意识认知加工过程独立存在, 独立运行。这一发现表明现有对心 智现象的融贯性要求并不完全合理, 无意识现象并非一定要与意识现象达成 某种解释上的一致才能被接受。 


\section{其次, 无意识认知加工具有不同于意识认知加工的特征}

1996 年 Sloman 就曾提出决策与推理双系统理论, 认为认知加工应该分 为快速、自动加工和慢速、规则加工两种形式[9]。Kahneman [10]、Evans [11] 在此基础上进一步提出了双系统作用模型：以直觉为基础的启发式系统 (heuristic system)和以理性为基础的分析式系统(analytic system)。前者快速、 不占用或很少占用心理资源, 过程无法察觉; 后者慢速、占用大量心理资源, 过程可以察觉。Dijksterhuis 等人为验证这一理论, 基于 “分心任务” 设计了 新的心理学实验来测试人类无意识思维加工和认知决策之间的关系, 并基于 实验结果提出了 “无意识思维理论(Unconscious Thought Theory, UTT)” [12] [13]。该理论核心观点为: 无意识认知加工过程明显不同于意识认知加工过程, 且在复杂任务决策时更加具有优势。

塞尔对无意识思维现象的解释显然与无意识思维理论不相符合。首先, 塞尔追求意识思维和无意识思维的一致性, 而无意识思维理论认为意识思维 和无意识思维独立存在, 并且无意识思维的容量更大, 关注的项目更多; 其 次, 塞尔认为意识思维与无意识思维的加工方式没有本质差异, 但无意识思 维理论认为意识思维容量有限, 可调动的认知资源也就有限, 所有加工只能 “自上而下” 展开, 无意识思维较少受到思维限制, 可调动更多认知资源, 因此加工可以 “自下而上” 展开; 再次, 塞尔认为意识思维在认识加工中占 据优势, 而无意识思维理论认为, 在处理复杂问题时, 由于受到认知资源和 注意等因素的影响, 意识思维可能会出现认知权重失衡的现象, 相比较而言, 无意识思维则会依据目标任务的自然权重自动评估各项认知因素的重要性, 从而在面对复杂情形时做出更恰当的决策; 最后, 塞尔认为思维规则具有普 遍性, 但无意识思维理论认为, 意识思维遵循较为严格的规则, 认知加工过 程更加严谨, 无意识思维不严格遵循规则, 而是采用关联加工的方式运行, 同时无意识思维运行时更加开放, 更易于生成创新观念。

鉴于塞尔对无意识心智状态的解释明显无法实现与上述认知科学实验研 究结果相融贯, 其论点理应受到更大的质疑。

\section{6. 小结}

塞尔对弗洛伊德的批评突出表现为他认为后者未能够实现解释上的融贯 性。为了纠正这一错误, 他提出了 “生物学的自然主义”, 并希望借助其中 的联结原则做出对无意识心智状态的合理解释。然而, 本文分析认为, 塞尔 同样没有将融贯性要求贯彻到底, 他对无意识状态的分类不合理, 对无意识 认识加工方式的解释也存在问题。

不仅如此, 鉴于塞尔视无意识心智状态为潜在的意识心智状态, 一旦其 对无意识心智状态的解释受到质疑, 我们有必要在后续研究中重新评价其对 意识心智状态的解释。

\section{Conflicts of Interest}

The authors declare no conflicts of interest. 


\section{References}

[1] 弗洛伊德. 弗洛伊德文集 $[M]$. 车文博, 主编. 长春: 长春出版社, 1998.

[2] 塞尔. 心灵的再发现 [M]. 北京: 中国人民大学出版社, 2005.

[3] 塞尔. 心灵导论 $[\mathrm{M}]$. 上海: 上海人民出版社, 2008.

[4] Chomsky, N. (1965) Aspects of Theory of Syntax. MIT Press, Cambridge. https://doi.org/10.21236/AD0616323

[5] Chomsky, N. (1975) Reflections on Language. Pantheon, New York.

[6] Smith, N. (2004) Chomsky: Ideas and Ideals. Cambridge University Press, Cambridge. https://doi.org/10.1017/CBO9780511606779

[7] 郭秀艳, 李林. 意识和无意识的关系——实证的视角 [J]. 心理学新探, 2006, 26(1): 3-8.

[8] Reber, A.S. (1967) Implicit Learning of Artificial Grammars. Journal of Verbal Learning \& Verbal Behavior, 6, 855-863. https://doi.org/10.1016/S0022-5371(67)80149-X

[9] Sloman, S.A. (1996) The Empirical Case for Two Systems of Reasoning. Psychological Bulletin, 119, 3-22. https://doi.org/10.1037/0033-2909.119.1.3

[10] Kahneman, D. and Frederick, S. (2002) Representativeness Revisited: Attribute Substitution in Intuitive Judgement. In: Gilovich, T., Griffin, D. and Kahneman, D., Eds., Heuristics and Biases. The Psychology of Intuitive Judgement, Cambridge University Press, Cambridge, 49-81. https://doi.org/10.1017/CBO9780511808098.004

[11] Evans, J.S. (2003) In Two Minds: Dual Process Accounts of Reasoning. Trends in Cognitive Sciences, 7, 454-459. https://doi.org/10.1016/j.tics.2003.08.012

[12] Dijksterhuis, A. (2004) Think Different the Merits of Unconscious Thought in Preference Development and Decision Making. Journal of Personality and Social Psychology, 87, 586-598. https://doi.org/10.1037/0022-3514.87.5.586

[13] Dijksterhuis, A., Maarten, W.B. and Nordgren, L.F. (2006) On Making the Right Choice: The Deliberation-without-Attention Effect. Science, 311, 1005-1007. https://doi.org/10.1126/science.1121629

\section{Appendix (Abstract and Keywords in Chinese)}

\section{对塞尔无意识心智状态解释的质疑}

摘要：基于 “生物学自然主义” 立场, 塞尔认为对心智现象的解释必须 与自然科学研究发现相融贯, 并提出了自己的 “联结原则”。本文对此提出 质疑。质疑主要从两个方面展开：无意识心智状态的分类、无意识认知加工 实验研究成果。论文分析发现, 首先, 塞尔无意识心智状态的分类不合理, 以语言知识为例, 塞尔将其视为深层无意识, 并进一步归属于非意识, 这与 语言运用实践和语言学研究发现不符; 其次, 塞尔对无意识认知加工方式的 解释有问题，他认为无意识心智状态仅是潜在的意识心智状态，两者的认知 加工方式理应一致, 这与认知实验研究发现不符。论文认为, 塞尔未达到自 己提出的融贯性要求。

关键词：无意识心智状态，联结原则，生物学自然主义，融贯性 